\title{
The Influence of the Volume Conductor on Electric Source Estimation
}

\author{
M.J. Peters* and H.J. Wieringa*
}

Key words: MEG; EEG; Inverse solution; Volume conduction.

\section{Introduction}

This tutorial concerns the influence of the head on the estimation of sources from MEG and EEG data.

The solution of the forward problem involves the calculation of the potential or magnetic field distribution at or near the outer surface of the volume conductor (i.e., the head), when the sources, the geometry and conductivities of the volume conductor are given. The estimation of electrical sources within the brain from the measured EEGs and/or MEGs is called the solution of the inverse problem. The solution of the inverse problem is not uniquely determined, different sources can produce similar electric potentials and magnetic fields. In order to solve the inverse problem, one has to make assumptions about the sources and the volume conductor in accordance with the electrophysiological reality. Since the neuronal response is slow compared with the time scales at which inductive and displacement currents occur, the electric field is conservative and all currents form closed loops. Consequently, part of the current resides outside the neurons. The extra-neuronal current, which is determined by the three-dimensional distribution of the electrical conductivity of the human head, contributes to the magnetic and electric field. However, our knowledge of this parameter is rather limited.

Algorithms are available to solve the inverse problem for both EEG and MEG using a head model consisting of an arbitrary number of concentric spheres, where the outer surface is locally fitted to the head. Forward

*University of Twente, Enschede, The Netherlands.

Accepted for publication: March 8, 1993.

Correspondence and reprint requests should be addressed to Dr. M.J. Peters, University of Twente, P.O. Box 217, 750 AE Enschede, The Netherlands.

Copyright (C) 1993 Human Sciences Press, Inc. problems have been solved for realistically shaped models. Algorithms to solve the inverse problem for realistically shaped models will be advantageous, because

A) the solutions will be more precise,

B) a locally fitting sphere is not uniquely defined, making EEG and MEG measurements susceptible of different interpretations, and

C) electrodes used for measuring EEG are not necessarily at the surface of the outer sphere, raising the problem: "Do we take the EEG measured by that electrode into account and/or should we take another sphere?"

Usually a patch of activity of the cortex is described by an equivalent current dipole. The inverse problem for a single current dipole is a six-parameter problem, in which three parameters determine the dipole's position and three its components. Normally, the estimation procedure is limited to the localization of the source. In other words only half of the parameters are calculated due to the fact that the volume conductor affects strongly the strength of the EEG based equivalent current dipole, and the MEG is only able to detect the tangential components. However, all six parameters can be determined more accurately if both MEG and EEG data are used. The strength of a source can be used to estimate the size of the active cortical layer.

The use of both MEG and EEG data can help us also to decide whether a single current dipole is enough to describe the measurements or that more sources have to be discriminated. Furthermore, MEGs and EEGs contain information which is partly independent. The noise in both measurements is also to a large extent uncorrelated and simultaneous electric and magnetic measurements have the highest information content (Pascual-Marqui and Biscay-Lirio 1991).

The MEG and EEG should preferably be used in com- 
bination with the MRI. Firstly, MRI scans can be used to derive a model of the head, which is necessary for the calculation of the location of electrical activity. Secondly, the MRI can be used to indicate this location within the head, thereby combining functional with structural information. Finally, the position of activity depicted in the MRI enables us to evaluate this result on physiological grounds, and if necessary, to correct the position within the error estimate.

\section{Basic equations}

In order to compute the electric potential and magnetic field generated by neural currents, it is sufficient to use the Maxwell equations in their quasi static form (Plonsey and Hepner 1967).

The total current density $\mathbf{j}$ is the sum of the primary current density $\mathbf{j}_{\mathrm{p}}$ and the ohmic volume current $\sigma \mathrm{E}$.

$$
\mathbf{j}=\mathbf{j}_{\mathrm{p}}+\sigma \mathrm{E}
$$

where $\sigma$ is the conductivity and $\mathrm{E}$ is the electric field.

The primary current density $\mathbf{j}_{\mathbf{p}}$ is only non-zero in the source region. Taking into account that charge does not accumulate in the tissue, we obtain the following equations:

$$
\begin{array}{r}
\Delta V=\left(\operatorname{div} j_{p}\right) / \sigma \\
\Delta B=-\mu_{0} \operatorname{rot} j
\end{array}
$$

where $\Delta$ is the Laplacian operator, $V$ is the electric potential, $\mu_{0}$ is the permeability of free space, and $B$ is the magnetic field.

These differential equations, the so-called Poisson equations, express the local relation between the current density at some point and the potential or magnetic field function in that immediate neighbourhood. Outside the volume conductor where there are no sources nor volume currents and outside the source regions of the volume conductor where the conductivity is uniform, these equations read:

$$
\Delta \mathrm{V}=0 \text { and } \Delta \mathrm{B}=0
$$

which are the so-called Laplace equations.

In electrostatics the potential due to a charge density in free space obeys Poisson's equation, $\Delta V=-\rho / \varepsilon_{0}$ (where $\varepsilon_{\mathrm{O}}$ is the permittivity of the free space) with solution

$$
V=\frac{1}{4 \pi \varepsilon_{o}} \int \frac{\rho d v}{r}
$$

according to Coulomb and the principle of superposition, where $\mathrm{r}$ is the distance between a source point and the point of observation and $\mathrm{dv}$ is an element of volume.

Analogous to the previously mentioned solution of Poisson's equation in electrostatics, we find as solution of the Poisson equation for $\mathrm{V}$ in a homogeneous volume conductor:

$$
V=\frac{1}{4 \pi \sigma} \int \frac{-d i v j_{p}}{r} d v
$$

A similar solution is found for the Poisson equation for $\mathbf{B}$. A similar integral equation is found in the case that the volume conductor is bounded and not homogeneous. Such an integral equation is non-local. All fundamental laws of electromagnetism can be formulated in two equivalent forms, one local and one non-local. From the volume integral equations surface integrals can be deduced (Barnard et al. 1967; Geselowitz 1970).

The equations mentioned above are solvable if all sources as well as the conductivity distribution are known. The uniqueness theorem states that if a solution of a potential problem within a given boundary is found, which reduces to the given potential distribution on that boundary, or to the normal derivative of the potential on that boundary, then this solution is the only correct solution of the potential equations within the boundary. This theorem provides justification for attempting any method of solution so long as the resulting solution can be shown to obey Laplace's equation in a source-free region and the Poisson equation in the source region. No matter how the solution is obtained, if it satisfies these conditions the problem is solved.

Actually only a few of the most idealized problems can be solved with some degree of simplicity. Nevertheless, the solution of problems with simple geometry is of value, because

A) it facilitates making the decision that it is worthwhile to seek the solution of more realistically shaped boundaries,

B) it furnishes a valuable check (rules of thumb)

C) it may show the dependency on parameters describing the volume conductor or source

D) some methods for solving the problem depend on prior knowledge of the general behaviour of the potential.

Some of these rules of thumb are: A dipole along the axis of symmetry of a volume conductor gives zero magnetic field outside the volume conductor. A tangentially oriented dipole within a conducting sphere gives a magnetic field with a radial component which is the same as the one generated by a dipole in a homogeneous medium. A dipole in the centre of a conducting sphere gives a potential at the surface of the sphere which is three times as large as the potential generated by the dipole in 


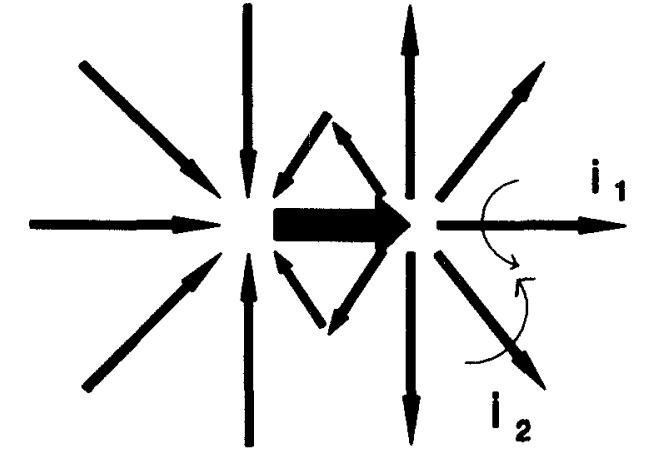

Figure 1.

a homogeneous medium of infinite extent. A dipole in a conducting half-space gives a potential at the interface which is twice the potential generated by the dipole in a homogeneous medium of infinite extent.

In this paper several methods to solve the forward problem will be discussed. In the case of conductors having complicated shapes, there are no analytical solutions. The only general method is to approximate the solutions by numerical methods.

Similarity with law of Coulomb: We start with the solution of the Poisson equation in the source region and a solution of the Laplace equation everywhere else for the case that the current dipole is embedded in a homogeneous conductive medium of infinite extent. We will use the fact that the field due to a point charge is similar to that due to a point current source.

The electric field generated by a point charge $Q$ in free space is according to the law of Coulomb

$$
E=\left(Q / 4 \pi \varepsilon_{0} r^{2}\right) \mathbf{e}_{r}
$$

where $r$ is the distance from the point charge to the point of observation, $\mathbf{e}_{\mathrm{r}}$ is a unit vector oriented along $\mathrm{r}$.

The current density due to a current source I, situated in a conducting homogeneous medium of infinite extent, is

$$
\mathbf{j}=\left(\mathrm{I} / 4 \pi \mathrm{r}^{2}\right) \mathbf{e}_{\mathbf{r}}
$$

and because according to Ohm's law $\mathbf{j}=\sigma \mathrm{E}$, it follows

$$
\mathbf{E}=\left(1 / 4 \pi \sigma \mathbf{r}^{2}\right) \mathbf{e}_{\mathbf{r}}
$$

Comparing the equations for $\mathbf{E}$, one notes that the equations are interchangeable if $\varepsilon_{\mathrm{O}}$ is replaced by $\sigma$, and Qby I.

From electrostatics we know that a dipole $p$ (i.e., a positive and a negative charge at a very short distance) gives rise to a potential

$$
\mathrm{V}=(\mathrm{p} \cos \Theta) / 4 \pi \varepsilon_{\mathrm{o}} \mathrm{r}^{2}
$$

where $\Theta$ is the angle between $\mathbf{p}$ and $\mathbf{e}_{\mathbf{r}}$.

Thus a current dipole $p$ (i.e., a current source and a current sink at a very short distance) will give rise to

$$
\mathrm{V} \infty=(\mathrm{p} \cos \Theta) / 4 \pi \sigma \mathrm{r}^{2}
$$

This expression is a solution of Poisson's equation in the source region and of Laplace's equation everywhere else.

The magnetic field due to a current dipole, situated in a homogeneous conducting medium of infinite extent, is according to the law of Biot and Savart

$$
\mathbf{B}=\mu_{0}(\mathbf{p} \times \mathbf{r}) / 4 \pi \mathbf{r}^{3}
$$

This equation shows that a current element generates a field which is perpendicular to the current element $\mathbf{p}$ and to the position vector $\mathbf{r}$, and is linearly proportional with $\sin \alpha_{0}$ where $\alpha_{0}$ is the angle between $p$ and $\mathbf{r}$.

The volume currents do not contribute to the field in this case as is demonstrated in figure 1, where current lines are drawn coming from the current source. For symmetry reasons it follows that the generated magnetic field $B$ is zero, because each current element like $i_{1}$ has a counterpart, here $i_{2}$, which produces an equal and opposite contribution to the magnetic field. Of course, the current that flows from the source must flow to the sink. The same holds for the volume currents due to a sink. The principle of superposition says that the resultant $B$ is simply the vector sum of the individual B's.

\section{Solution by the method of images}

An overview of all solutions by means of the method of images which can be of interest for EEG is given in figure 2 .

In the first column the actual systems for A, B and C are depicted while equivalent systems are presented in the second and third columns.

The actual system shown in figure $2 \mathrm{~A}$ consists of two dipoles situated in a conducting half space with conductivity $\sigma_{1}$ bounded by a non-conductor. The interface is the $\mathrm{z}=0$ plane. This system is for the region $\mathrm{z}<0$ equivalent to a space of infinite extent with conductivity $\sigma_{1}$ and both dipoles $\mathbf{p}_{1}$ and $\mathbf{p}_{2}$ have mirrored ones $\mathbf{p}_{1}{ }^{\prime}$ and $\mathbf{p}_{2}{ }^{\prime}$ being otherwise the same as the originals.

The actual system shown in figure $2 \mathrm{~B}$ consists of two dipoles situated in a conducting half space with conductivity $\sigma_{1}$ bounded by a conductor with conductivity $\sigma_{2}$. This system is for the region $\mathrm{z}<0$ equivalent to a space of infinite extent with conductivity $\sigma 1$. The dipoles $p_{1}$ and $p_{2}$ have mirrored ones $p_{1}{ }^{\prime}$ and $p_{2}{ }^{\prime}$ where $p_{1}{ }^{\prime}=\left(\sigma_{1^{-}}\right.$ 


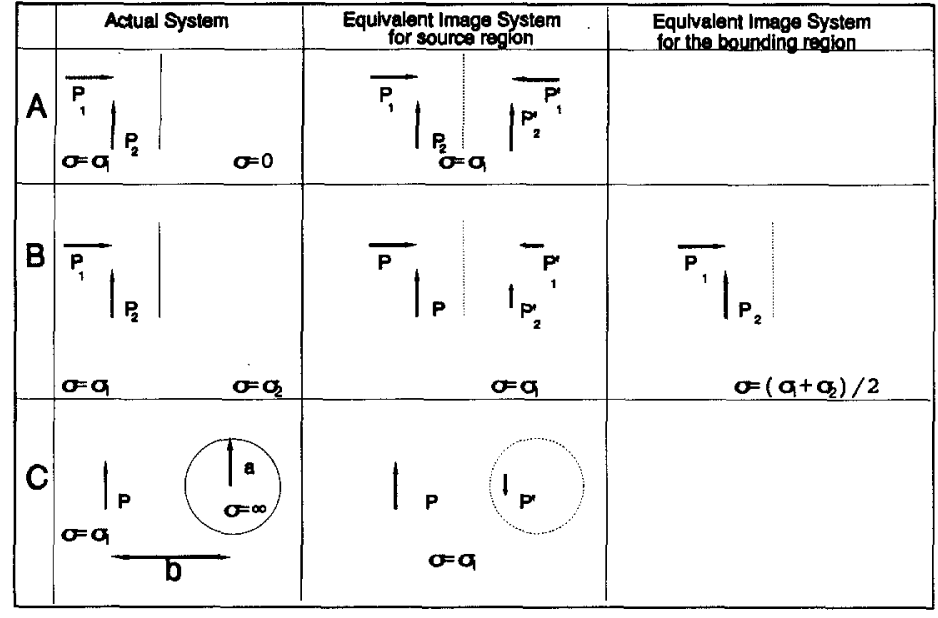

Figure 2.

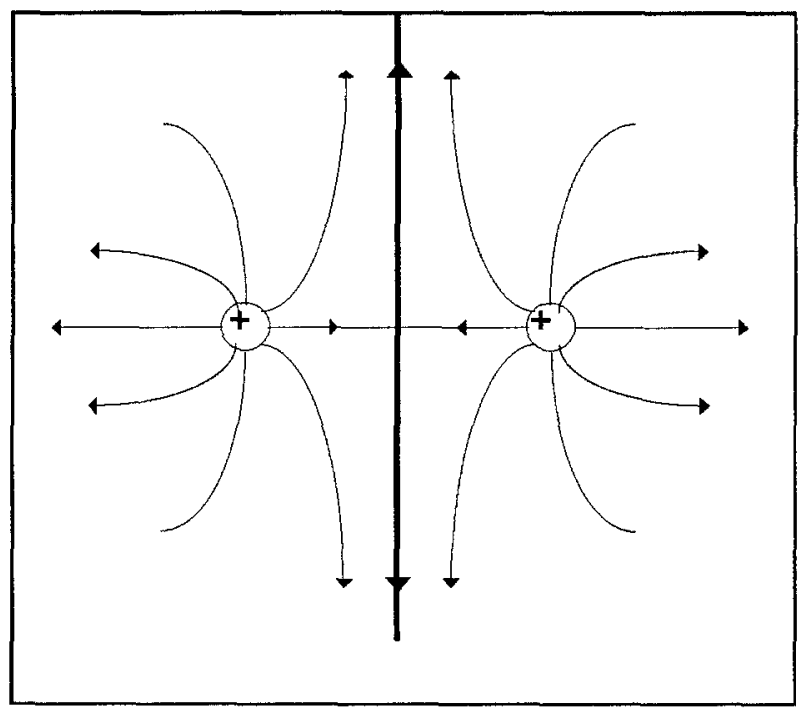

Figure 3.

same current source in an infinite homogeneous medium.

If we add a sink and apply the same reasoning then we see that for a current dipole embedded in a homogeneous conductive half space the potential on the boundary is given by:

$$
\mathrm{V}=2 \mathrm{~V}_{\infty}
$$

The sphere with $\sigma=\infty$ can be looked upon as a very rough model of a lesion (edema). Even using such a rough model it is easy to understand why magnetic flux reversal can occur as described by Ueno and Iramina (1990). An example is sketched in figure 4. A current dipole $\mathbf{p}$ which is almost radially oriented within a sphere gives in point $\mathrm{A}$ a field which enters the paper. If an edema is situated between the dipole and the surface of the sphere, the image source $\mathbf{p}^{\prime}$ will be oriented more tangentially. The field in A due to $\mathbf{p}^{\prime}$, when calculated using the law of Biot and Savart, dominates the field due to $p$ because $p^{\prime}$ is nearer to $A$ and also has a larger tangential component. Consequently, the field in $\mathrm{A}$ is reversed.

\section{Method of axial expansion}

The method of axial expansion can be used for determining the external potential or field of axially symmetric volume conductors. The sources have to be on an axis of symmetry and the potential or field on that axis has to be known. We will show, by means of this method, that if a current dipole is oriented along the axis of such a volume conductor, the total magnetic field outside the at the surface which would have been generated by the 


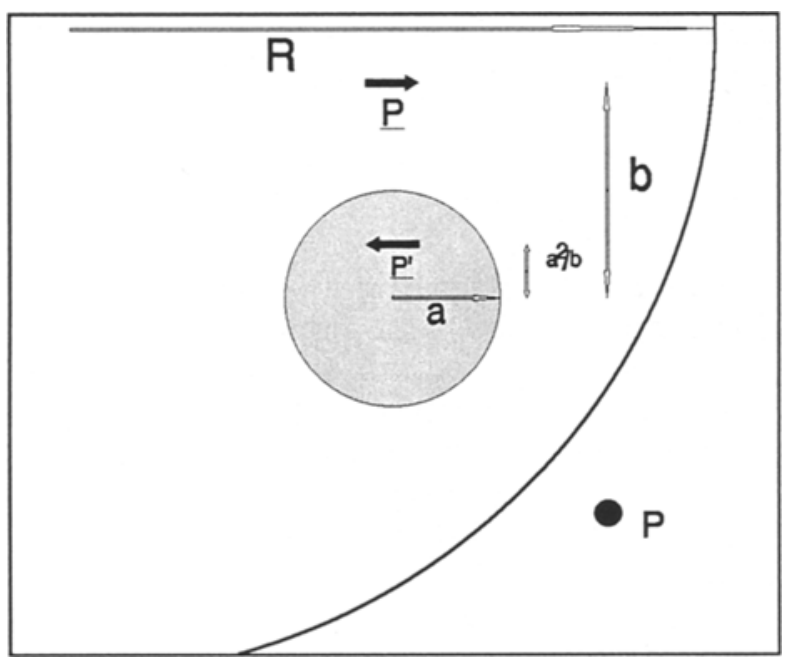

Figure 4. Reversal of B-field due to an edema.

volume conductor will be zero.

The current dipole causes a magnetic field, which is perpendicular to $\mathrm{p}$ and $\mathbf{e}_{\mathrm{r}}$, and linearly proportional with $\sin \alpha$, where $\alpha$ is the angle between $\mathbf{p}$ and $\mathbf{e}_{\mathrm{r}}$. As a consequence the source does not contribute to the magnetic field on the axis outside the volume conductor $(\alpha=$ $0)$. The contributions to the magnetic field on the axis due to the current line elements $a$ and $b$ just cancel and so do all other pairs (see figure 5).

B outside the volume conductor, where there are no sources nor ohmic currents, is given by the Laplace equation, $\Delta \mathbf{B}=0$. The solution of each component of the magnetic field can be represented by so-called spherical harmonics. For $\mathbf{B}_{\mathrm{r}}$ the solution reads:

$$
B_{r}=\sum_{n=0}^{\infty}\left(A_{n} r^{n}+\frac{B_{n}}{r^{n+1}}\right) P_{n}(\cos \theta)
$$

where $\Theta$ is measured with respect to the symmetry axis.

The fact that these so-called spherical harmonics are indeed solutions of the Laplace equation can be verified by direct substitution. Since on the axis $\Theta=0$, it follows that $P_{n}=1$ for all $n$, and $B_{r}$ on the axis is

$$
B_{r}=\sum_{n=0}^{\infty} A_{n} Z^{n}+\frac{B_{n}}{Z^{n+1}}
$$

where $\mathrm{z}$ is the distance from the origin along the axis. However, $\mathbf{B}$, and consequently also $B_{r}$, is zero on the axis as was demonstrated above. The coefficients $A_{n}$ and $B_{n}$ in equation 2 therefore must be zero and can be substituted in equation 1 . So from $B_{r}$ on the axis, it follows

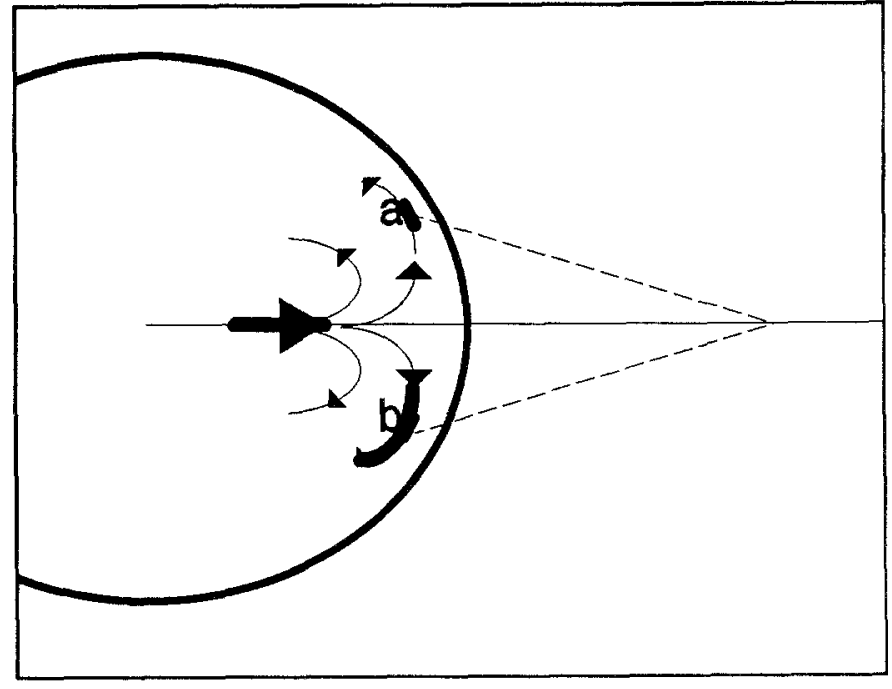

Figure 5.

that for all points of the space outside the volume conductor $B_{r}$ is zero. The same is true for the tangential components of the magnetic field.

Thus we found that if a current dipole is oriented along the axis of a volume conductor, which has axial symmetry, the total magnetic field everywhere outside the volume conductor is zero. An example is a radial current dipole in a volume conductor consisting of concentric spheres. The conductivity in the various compartments may be anisotropic in the sense that the conductivity in the radial direction may differ from that in the tangential directions.

An other example is a homogeneous current dipole layer in the shape of a circular disk where the normal vector on the disc is radially oriented. Also in this case we find that outside a volume conductor with axial symmetry the magnetic field is zero.

The method of axial expansion can be used also to calculate the potential generated by a circular dipole layer of radius a and dipole density $\tau$.

On the axis $\mathrm{V}$ is obtained by dividing the disk in infinite small rings of radius $R$ and width $d R$ (see figure 6):

$$
\begin{aligned}
V & =\frac{1}{4 \pi \sigma} \int_{0}^{a} \frac{\tau \cos \alpha 2 \pi R d R}{x^{2}} \\
& =\frac{\tau}{2 \sigma}\left(\frac{z}{\sqrt{a^{2+} z^{2}}}-1\right)
\end{aligned}
$$

Expanding the potential in the power series of $z$ and noting that the total dipole strength of the disk is $\mathrm{p}=\tau \pi \mathrm{a}^{2}$ we obtain 


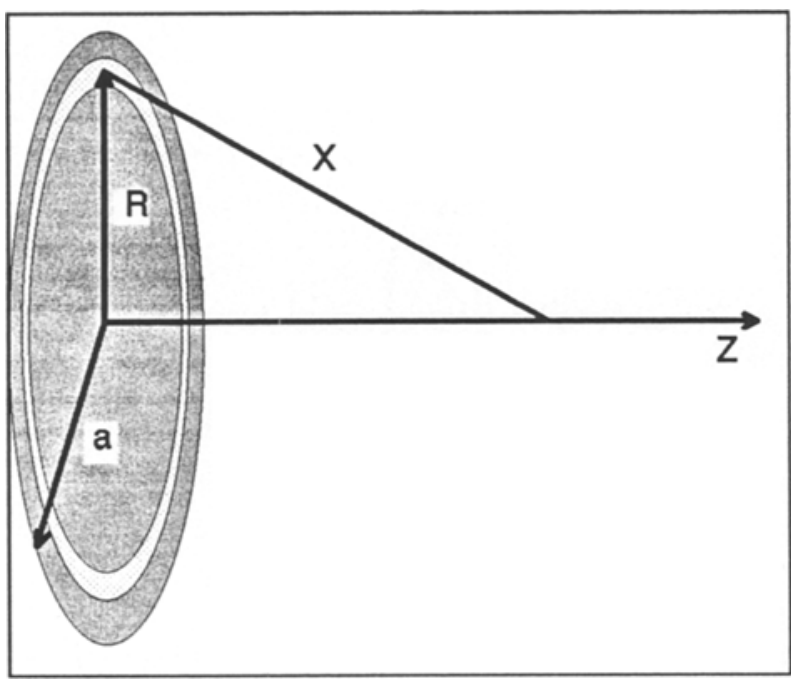

Figure 6.

$$
\begin{gathered}
V=\frac{p}{2 \pi a^{2} \sigma}\left[\left(1+\frac{a^{2}}{z^{2}}\right)^{-1 / 2}-1\right] \\
=\frac{P}{4 \pi \sigma z^{2}}\left(1-\frac{3 a^{2}}{4 z^{2}}+\frac{15 a^{4}}{24 z^{4}}+\ldots\right)
\end{gathered}
$$

The relative error resulting from using an equivalent dipole in the centre of the disk instead of the dipole layer is in second order approximation smaller than $3 \%$ for $z$ $>5 \mathrm{a}$. This formula expresses the potential on the axis and gives for $A_{n}$ and $B n$ of equation 1

$A_{n}=0, B_{0}=0, B_{1}=\frac{p}{4 \pi \sigma}, B_{2}=0, B_{3}=-\frac{3 a^{2} p}{16 \pi \sigma}$, etc

The complete potential is therefore

$$
V=\frac{p}{4 \pi \sigma z^{2}}\left(1-\frac{3 a^{2}}{4 z^{2}} P_{3}(\cos \theta)\right)
$$

\section{Methods of harmonics}

This method is used to derive equations for the potential and magnetic field generated by a current dipole within a volume conductor consisting of concentric spheres, confocal ellipsoids, and eccentric spheres (De Munck 1988; Cuffin 1977; Cuffin 1991). The number of concentric spheres is not restricted and the conductivity in the radial direction may differ from that in the tangential ones.

The potential distribution at the surface due to a dipole in a homogeneous sphere can be expressed by a closed formula as derived by Franck (1952). The potential somewhere else in the sphere and the potential based on more complicated spherical models of the head can not be expressed in a closed-form but will contain an infinite series of summations. To perform a series summation is rather time consuming and therefore several groups use the expression for the potential at the surface of a homogeneous sphere and make corrections so that effectively one uses a model consisting of a sphere surrounded by two concentric shells representing the skull and the scalp (Ary et al. 1981).

We will demonstrate the method of harmonics for a simple case: a current dipole in the centre of a sphere with radius $R$, oriented along the $z$-axis. Because there is axial symmetry the solution Laplace's equation in spherical coordinates, is given by:

$$
V(r, \theta)=\sum_{n=0}^{\infty}\left(A_{n} r^{n}+\frac{B_{n}}{r^{n+1}}\right) P_{n}(\cos \theta)
$$

The arbitrary constants in this formula have to be adjusted to satisfy the boundary conditions.

The boundary conditions are: For $r$ approaching 0 the solution is $(p \cos \theta) / 4 \pi \sigma r^{2}$. For $r=R$ the normal component of the current density is zero:

$$
\partial \mathrm{V} / \partial \mathbf{r}=0
$$

The first boundary condition leads to $\mathrm{B}_{0}=0 ; \mathrm{B}_{1}=$ $p / 4 \pi \sigma$ (because $\left.P_{1}(\cos \Theta)=\cos \Theta\right) ; B_{k}=0$ for $k>1$.

These findings are substituted in the second boundary condition, which requires that for each value of $\Theta$ :

$$
-\frac{2 p \cos \theta}{4 \pi \sigma r^{3}}+\sum_{n=0}^{\infty} n A_{n} r^{n-1} P_{n}(\cos \theta)=0
$$

implying that $\mathrm{A}_{0}=$ unknown, and will be chosen zero because one can add to $\mathrm{V}$ any quantity that is independent of the coordinates without affecting the currents. $A_{1}=2 p /\left(4 \pi \sigma R^{3}\right) . A_{k}=0$, for $k \geq 2$.

Thus for $\mathrm{r} \leq \mathrm{R}$

$$
V=\frac{p \cos \theta}{4 \pi \sigma r^{2}}+\frac{p 2 r \cos \theta}{4 \pi \sigma R^{3}}
$$

This expression says that the potential at the surface of the sphere generated by a current dipole in the centre is

$$
\mathrm{V}=3 \mathrm{~V}_{\infty}
$$

The same method can be used to obtain the expression of $\mathrm{V}$ generated by a radially oriented current dipole on 


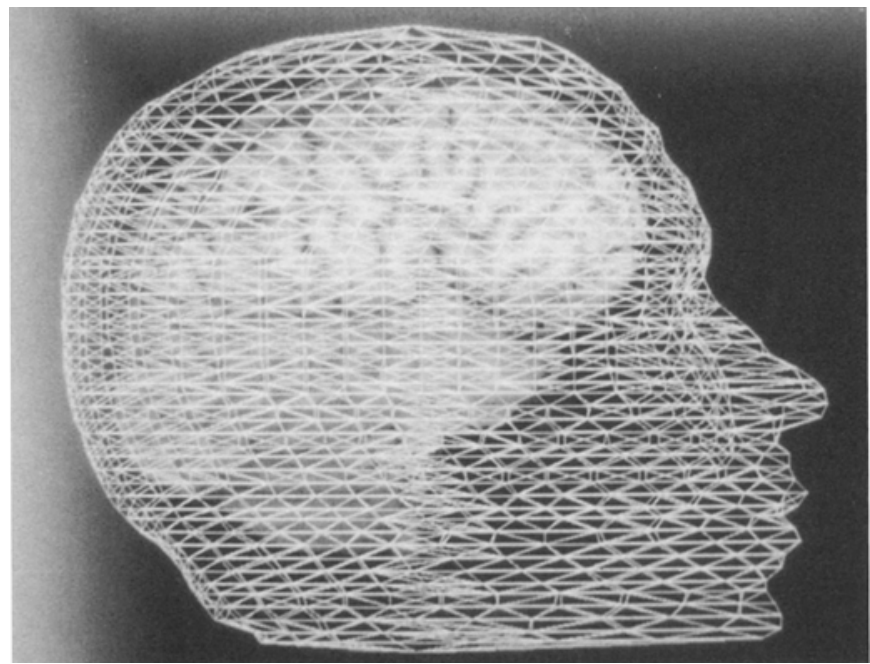

Figure 7. Triangulated surface of the head with a 3D view of the brain surface.

an axis. When the dipole has a different orientation and there is no longer axial symmetry, we have to replace equation 1 by a more complicated one which can be found in books on electromagnetism.

\section{Boundary element method}

The boundary element method has as a main characteristic that it is based upon the reformulation of the volume integral in a surface integral. This method is used in a so-called compartment model, in which the electric conductivity is assumed to be homogeneous and isotropic in each of the distinguished compartments.

A piecewise homogeneous volume conductor can be considered equivalent to a uniform conducting medium of infinite extent in which fictitious sources (the so-called secondary sources) lie at the interfaces between regions of different conductivity, the outer surface included. The orientation of these secondary sources is normal to the interfaces and their strengths are proportional to the local potential and the conductivity difference of the successive compartments. The secondary sources add to the primary sources to set up the observed magnetic field and electric potential. To obtain a set of algebraic functions from the surface integral equation, the surfaces are triangulated or paneled. The potential is considered to be constant or to vary linearly over a triangle.

The most comprehensive realistically shaped model consisted of four realistically shaped compartments representing the brain, the cerebrospinal fluid, the skull and the scalp (Meijs 1988). The number of triangles of each compartment boundary was of the order of 600 . Because the rate of change of the potential is increased if the distance of the source to the triangle is decreased, the areas of the triangles were chosen smaller in the section of the head where the sources were postulated. Consequently, the accuracy of the results were enhanced due to this local refinement of the triangular paneling of the boundaries.

The advantage of using the boundary element method is evident, it requires only a discretization of a few surfaces instead of a discretization of the whole volume of the head. The triangulated surfaces can be automatically generated from MRI scans (see figure 7).

\section{Finite element method}

In order to assess the importance of anisotropy in the conductivity, to study the effect of scalpless regions such as the eyes, and to estimate the effect of complex-shaped fluid-occupied volumes such as the ventricles, it is necessary to perform volume integration. Three-dimensional finite elements, such as tetrahedrons or cubes, can be used in order to subdivide the relevant volumes for the purpose of integration. Although this method is one of the most successful methods for numerically solving potential differential equations, it was only recently used to solve volume conduction problems (Bertrand et al. 1991; Yan 1991).

\section{The influence of the volume conductor}

The conductivities of the various tissues within the head play a significant role in the volume conduction problem. Measurements of the conductivity are difficult to carry out, because in postmortem or biopsy samples it will be influenced by necrosis and fluid loss. Values of the conductivities can be adapted from Geddes and Baker (1967), who collected values of the specific conductivities of biological materials. Averaged values of the conductivities, which are usually taken, are for the brain $0.33 \mathrm{Sm}^{-1}$; for the cerebrospinal fluid $1.0 \mathrm{Sm}^{-1}$; for the skull $0.0042 \mathrm{Sm}^{-1}$, and for the scalp $0.33 \mathrm{Sm}^{-1}$. Cohen and Cuffin (1983) compared MEG and EEG maps, showing a dipole-like pattern, based on the N20 signal of the scalpevoked response following stimulation of the median nerve. The MEG and EEG were measured under the same experimental conditions. They concluded that for their sources, the head appears to behave as a model consisting of concentric spheres for both MEG and EEG. In order to explain the MEG and EEG with the same current dipole, they assigned values for the conductivity to the tissues, being in the range of values as given by Geddes and Baker (1967). These values were for the brain, fluid, skull and scalp 0.46, 1.4, 0.0058 and $0.46 \mathrm{Sm}^{-1}$, respectively.

Algorithms are available to solve the inverse problem for both MEG and EEG using a head model consisting of 
an arbitrary number of shells. A dipole can be found for which the model predicted distribution of $\mathbf{B}$ and/or $V$ is closest to the measured distribution in least square error sense. Varying data for the radii and conductivities within the values given in the literature leads to changes in the localization based on EEG of the order of $0.5 \mathrm{~cm}$, to changes in the strengths of $100 \%$ and to changes in the orientation of $0.5 \%$ (Stok 1988; Peters and De Munck 1990). The fact that the orientation of the dipole is preserved (errors of the order of $0.5 \%$ ) was also found by Cuffin et al. (1991), who studied the influence of the real head by means of implanted electrodes.

Within the context of spherical symmetric model, the MEG is independent of the conductivities involved. The MEG is only dependent on the choice of the centre of the sphere and on uncertainties in the position of the pick-up coils of the magnetometer with respect to the head. This leads to possible errors in the localization of the order of a few mm (Buchanan 1989). The uncertainty in the choice of the centre of the sphere can be avoided by using a realistically shaped model of the head.

As a consequence we can use MEG measurements: 1) to localize tangentially oriented current dipoles and 2) to estimate their strengths. We can use the EEG: 1 ) to decide if there are also radially oriented sources, 2 ) to localize the radially oriented sources, and 3) to compute the orientation of the dipoles which were also found by MEG.

If we know the tangential components plus the orientation we know the strength. The strengths of the dipoles, which are deduced from the combination MEG and EEG, are now rather precisely known.

Simulations using a realistically shaped model of the head carried out by the boundary element method lead to the following conclusions (Meijs et al. 1985; Bin He et al. 1987; Hämäläinen and Sarvas 1989): a. The influence of the model used increases with source depth. b. The influence of the nonsphericity of the models on the MEG is negligible for sources at a depth of $20 \mathrm{~mm}$ from the scalp. c. Clear differences are found in fronto-temporal and frontal areas at a depth of more than $20 \mathrm{~mm}$ and for sources close to the irregularity shaped bottom of the skull. $d$. The influence of the model used on the EEG is larger than on the MEG. e. The direction of the dipoles is practically always retained.

The strengths of the dipoles were found to be dependent on the local thickness of the various compartments, making it difficult to decide if the strengths were influenced.

\section{Discussion}

Once the dipoles are estimated they can be depicted in an MRI data-set. The electrical active cortical layer can be estimated from the strength of the equivalent dipole if the transcortical current density is known. According to Freeman $(1975$, p. 254) the current density is about 270 $n A m m^{-2}$.

The electric potential and the magnetic field for any homogeneous dipole layer with a circular rim (e.g., a disc, a hemisphere or a homogeneous dipole layer having the shape of a half prolate spheroid) can be approximated by a single current dipole at the centre of the circle of the ground plane, at distances comparable to the dimensions of the layer. Orientations of both the tangential dipole obtained from MEG and the one from EEG, the strengths and positions have to be in accordance with volume conduction theory and electrophysiologic and anatomical findings. MRI images will help us to evaluate the findings. An example of a source obtained is given in figure 8 .

\section{References}

Ary, J.P., Klein, S.A. and Fender, D.H. Location of sources of evoked scalp potentials: corrections for skull and scalp thicknesses. IEEE Trans. on Biomed. Eng., 1981, BME-28: 447-452.

Barnard, A.C.L., Duck, J.M., Lynn, M.S. and Timlake, W.P. The application of electromagnetic theory to electrocardiology II. Biophys. J., 1967, 7: 463-491.

Bertrand, O., Thevenet, M. and Perrin, F. 3D Finite element method in brain electrical activity studies. In: J. Nenonen, H.M. Rajala and T. Katila (Eds.), Biomagnetic localization and 3D modeling. Otaniemi, 1991.

Bin He, Musha, T., Okamoto, Y., Homma, S., Nakajima, Y. and Sato, T. Electric dipole tracing in the brain by means of the boundary element method and its accuracy. IEEE Trans. on Biomed. Eng., 1987, BME-34: 406-414.

Buchanan, D.S. Impact of different noise sources on dipole localization in the spherical model. In: S.J. Williamson, M. Hoke, G. Stroink, M. Kotani (Eds.), Advances in Biomagnetism. Plenum Press, New York, 1989: 539-542.

Cohen, D. and Cuffin, B.N. Demonstration of useful differences between magnetoencephalogram and electroencephalogram. Electroenceph. Clin. Neurophysiol., 1983, 56: 38-51.

Cuffin, B.N. and Cohen, D. Magnetic fields of a dipole in special volume conductor shapes. IEEE Trans. on Biomed. Eng., 1977, BME-24: 372-381.

Cuffin, B.N. Eccentric spheres models of the head. IEEE Trans. on Biomed. Eng., 1991, BME-38: 871-878.

Cuffin, B.N., Cohen, D., Yunokuchi, K., Maniewski, R., Purcell, C., Cosgrove, G.R., Ives, J., Kennedy, J. and Schomer, D. Tests of EEG localization accuracy using implanted sources in the human brain. Annals of Neurology, 1991, 29: 132-138.

De Munck, J.C. The potential distribution in a layered anisotropic spheroidal volume conductor. J. Appl. Phys., 1988, 64(2): 464-470.

Frank, E. Electric potential produced by two current sources in a homogeneous conducting sphere. Journal of Appl. Physics, 1952, 23: 1225-1228.

Freeman, W.J. Action in the nervous system. Academic Press, 
New York, 1975.

Geddes, L.A. and Baker, L.E. The specific resistance of biological materials- A compendium of data for the biomedical engineer and physiologist. Med. Biol. Eng., 1967, 5: 271-293.

Geselowitz, D.B. On the magnetic field generated outside an inhomogeneous volume conductor by internal sources. IEEE Trans. on Magn., 1970, 6: 346-347.

Hämäläinen, M.S. and Sarvas, J. Realistic conductivity geometry model of the human head for the interpretation of neuromagnetic data. IEEE Trans. on Biomed. Eng., 1989, BME-36: 165-172.

Meijs, J.W.H., Peters, M.J., Oosterom, A. van. Computation of MEGs and EEGs using realistically shaped multi-compartment model of the head. Med. Biol. Engng. Comput., 1985, 23 suppl. part 1: 36-37.

Meijs, J.W.H. The influence of head geometries on electro-and magnetoencephalograms. Ph. D. Thesis, University of Twente, The Netherlands, 1988.

Pascual-Maqui, R.D. and Biscay-Lirio, R. 8th International Conference on Biomagnetism, Munster, 1991.

Plonsey, R. and Heppner, D.B. Considerations of quasi-stationary in electrophysiological systems. Bulletin of Mathematical Biophysics, 1967, 29: 657-664.

Stok, C.J. The influence of model parameters on EEG/MEG source estimation. IEEE Trans. on Biomed. Eng., 1987, BME34: 289-296.

Ueno, S. and Iramina, K. Modeling and source localization of MEG activities. Brain Topography, 1990, 3: 151-165.

Yan, Y., Nunez, P.L. and Hart, R.T. Finite-element model of the human head: scalp potentials due to dipole sources. Med. Biol. Eng. Comp., 1991: 475-481. 